resistance change of selenium with rise of temperature. The color change of the crystals points to some sort of a transformation. G. F. S.

The Age of the Earth. (Nature, Oct. 13, I921.)-A discussion on this subject was held at the recent meeting of the British Association for the Advancement of Science at Edinburgh at which the points of view of physics, geology and astronomy were presented. Lord Rayleigh arrived at the age of uranium-bearing rocks from a study of their amounts of uranium-lead and helium. The rates of transformation from uranium through all the intermediate steps klown to lead are known. This is not ordinary lead but lead of atomic weight 206. Assuming that there has been no change in the rates of transformation an age of $925,000,000$ years is derived from these rocks. Considerations based on the helium content lead to concordant results. He assigns a period of a few billion years as the duration of the earth's crust in a condition fit for human habitation.

The geologists did not present an undivided front in respect to the proper interpretation of the data of their science. Professor Gregory held that prevalent age estimates might well be multiplied by ten or twenty. Results thus obtained would agree with those emanating from the field of physics.

Eddington from considerations based on certain variable stars concluded that Lord Kelvin's famous time-scale must be multiplied by 700 . Jeffreys used two entirely distinct lines of approach, temperature distribution downward in the earth's crust and the evolution of the solar system by tides. In both cases he derives about two billion years as the length of time since the solidification of the crust.

G. F. S.

\title{
An Anticipation of One of the Results of Einstein's Theory
} of Relativity. P. Lenard. (Ann. d. Physik, Number 15, I92 I.) Einstein predicted that a ray of light from a star would be deviated from its path when it passes near the sun. This was confirmed by photographs of stars taken a couple of years ago at the time of a total eclipse of the sun. Professor Lenard, who is, to say the least, not strongly prejudiced in favor of the theory associated with the name of Einstein, takes pleasure in directing attention to a forgotten article, published by J. Soldner in I8or. The latter was a German mathematician, born in Bavaria, the son of a peasant. According to Professor Lenard's somewhat caustic comment, "He had besides the advantage of not having attended too many schools." In the article mentioned this scientist of Napoleonic times attributed to a ray of light all the absolute properties of matter and considered it as subject to gravitation. On this basis he calculated the deviation such a ray would experience in passing near the sun and arrived at an expression which Einstein likewise reached more than a century later from considerations of the Relativity Theory. 